
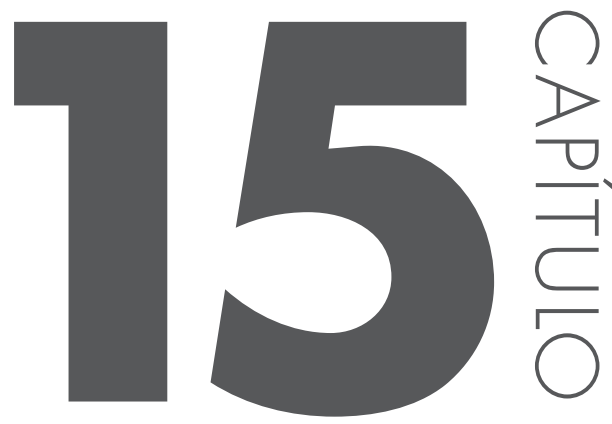

\title{
O PERFIL DA MORTALIDADE DOS TRABALHADORES PAULISTAS (2000-2010)
}

Luis P Ortiz Flores ${ }^{1}$

1 Professor Titular do Departamento de Atuária e Métodos Quantitativos/FEA/PUCSP e Analista de Projetos da Fundação Sistema Estadual de Análise de Dados - SEADE.

\section{RESUMO}

As estatísticas sobre a população economicamente ativa ajudam a entender importantes aspectos socioeconômicos, servindo de base para formular programas públicos. Tais informações compreendem o potencial de mão de obra com que pode contar o setor produtivo, isto é, a população ocupada e a desocupada. Este artigo traça o perfil da vida ativa dos trabalhadores paulistas, considerando seus fatores condicionantes, de forma a ressaltar dados de relevo para a formulação de políticas públicas. São utilizadas informações dos censos demográficos realizados pelo IBGE em 2000 e 2010 e as estatísticas de óbitos 
provenientes do Sistema de Estatísticas Vitais, elaboradas pela Fundação Seade. Calculam-se as taxas de atividade brutas e específicas, por sexo e idade, e o número de anos de vida ativa, utilizando as tábuas de mortalidade construídas para esses anos. São também estimadas a esperança de vida aos dez anos de idade, na hipótese de eliminar grupos de doenças classificadas como evitáveis, e sua repercussão no tempo de permanência na atividade econômica. Se for possível reduzir e/ou eliminar as doenças não transmissíveis e as causas externas, o diferencial segundo gênero da mortalidade seria reduzido significativamente, bem como aumentaria expressivamente a esperança de vida aos dez anos de idade e, consequentemente, o tempo de permanência na atividade econômica, especialmente para a população masculina.

Palavras-chave: população economicamente ativa. Taxas de participação. Mortalidade. Tendência da mortalidade.

Causas de morte 


\section{INTRODUC̣ÃO}

As estatísticas sobre a população economicamente ativa (PEA) ${ }^{2}$ são utilizadas no esclarecimento de importantes aspectos da vida econômica e social e na formulação de medidas para assegurar a melhoria das suas condições, servindo de base para formular programas de desenvolvimento econômico e social e possibilitando avaliar o melhor investimento dos recursos, bem como as mudanças que poderiam ocorrer em tais circunstâncias.

Estas informações compreendem o potencial de mão de obra com que pode contar o setor produtivo, isto é, a população ocupada e a desocupada. $\mathrm{O}$ presente artigo traça o perfil da vida ativa dos trabalhadores paulistas, considerando seus fatores condicionantes, de forma a ressaltar dados de relevo para a formulação de políticas públicas. Com esse fim, são exploradas as características demográficas da população economicamente ativa no Estado de São Paulo, em especial a influência da mortalidade, segundo gênero, idade e causas de morte, no tempo de permanência na vida ativa dessa população.

A oferta de mão de obra é definida como o número potencial de trabalhadores disponível para a produção de bens e serviços econômicos de uma sociedade. $\mathrm{O}$ número de pessoas que poderiam participar na atividade econômica é determinado pelo tamanho da população e por sua estrutura segundo sexo e idade. Essas características, por sua vez, estão relacionadas às tendências das variáveis demográficas: fecundidade, mortalidade e migração. Também exerce considerável influência no tamanho da população economicamente ativa a situação econômica do país em determinado momento, que pode ter efeito, por exemplo, no chamado desalento ${ }^{3}$.

No Estado de São Paulo, no período 2000-2010, em torno de 55\% da população de dez anos e mais foi considerada economicamente ativa (PEA). Entre os homens, essa proporção tem se mantido praticamente constante em $70 \%$; já entre as mulheres, registra-se um significativo incremento, chegando a $52 \%$, em 2010.

Paralelamente, observam-se mudanças significativas na dinâmica demográfica paulista, em especial uma diminuição acentuada no ritmo de crescimento, como resultado da queda da fecundidade - em 2010, a taxa atingiu 1,7 filho por mulher - da longevidade crescente, chegando a 71,5 anos para os homens e 78,6 para as mulheres, e da participação reduzida da migração, com uma taxa de somente 1,2 migrante ao ano por mil habitantes (WALDVOGEL et. al., 2012).

\footnotetext{
${ }^{2} \mathrm{~A}$ PEA é composta por pessoas de 10 a 65 anos de idade que foram classificadas como ocupadas ou desocupadas na semana de referência da pesquisa.

${ }^{3}$ Dá-se o nome de desalento ao fenômeno no qual as pessoas não economicamente ativas que estavam procurando emprego há pelo menos seis meses desistem por não encontrarem qualquer tipo de trabalho, ou um trabalho com remuneração adequada, ou ainda um trabalho de acordo com as suas qualificações.
} 
Estima-se que, nos últimos 50 anos, a esperança de vida ao nascer teve um aumento maior entre as mulheres (14,9 anos) do que entre os homens (12,4 anos). No período mais recente, o ganho foi maior na população masculina, com incremento de 4,2 anos, do que na feminina, que registrou ampliação de 2,4 anos. Assim, a diferença da esperança de vida ao nascer entre homens e

\section{TABELA 1}

Esperança de vida ao nascer e esperança de vida aos 10 anos de idade, segundo sexo

Estado de Săo Paulo - 1960-2010

\begin{tabular}{|c|c|c|c|c|c|c|}
\hline \multirow{2}{*}{ Sexo } & \multicolumn{4}{|c|}{ Esperança de Vida ao nascer } & \multicolumn{2}{|c|}{ Ganhos (anos) } \\
\hline & 1960 & 1980 & 2000 & 2010 & $1960-2010$ & 2000-2010 \\
\hline Homens & 59,0 & 63,3 & 67,2 & 71,4 & 12,4 & 4,2 \\
\hline Mulheres & 63,7 & 70,0 & 76,2 & 78,6 & 14,9 & 2,4 \\
\hline Diferença & 4.7 & 6.7 & 9,0 & 7,2 & & \\
\hline \multirow{2}{*}{ Sexo } & \multicolumn{4}{|c|}{ Esperança de Vida aos 10 anos de idade } & \multicolumn{2}{|c|}{ Ganhos (anos) } \\
\hline & 1960 & 1980 & 2000 & 2010 & $1960-2010$ & $2000-2010$ \\
\hline Homens & 56,1 & 57,8 & 58,8 & 62,6 & 6,5 & 3,8 \\
\hline Mulheres & 60,3 & 64,0 & 67,6 & 69,7 & 9,4 & 2,1 \\
\hline Diferença & 4,2 & 6,2 & 8,9 & 7,0 & & \\
\hline
\end{tabular}

Fonte: IBGE - Censo Demográfico de 2000 e 2010; Fundaçăo Seade.

TABELA 2

Taxas de mortalidade por sexo e grupos de idades

Estado de Săo Paulo - 2000 e 2010

\begin{tabular}{|c|c|c|c|c|c|c|}
\hline \multirow{2}{*}{$\begin{array}{c}\text { Grupos de } \\
\text { idades }\end{array}$} & \multicolumn{3}{|c|}{2000} & \multicolumn{3}{|c|}{2010} \\
\hline & Homens & Mulheres & ISMM (*) & Homens & Mulheres & ISMM (*) \\
\hline $\begin{array}{l}10 \text { a } 14 \\
15 \text { a } 19\end{array}$ & $\begin{array}{c}4,7 \\
23,9\end{array}$ & $\begin{array}{l}2,8 \\
5,1\end{array}$ & $\begin{array}{l}1,6 \\
4,7\end{array}$ & $\begin{array}{c}3,1 \\
11,5\end{array}$ & $\begin{array}{l}2,9 \\
5,6\end{array}$ & $\begin{array}{l}1,1 \\
2,0\end{array}$ \\
\hline 20 a 24 & 35,2 & 6,4 & 5,5 & 17,8 & 6,3 & 2,8 \\
\hline 25 a 29 & 36,3 & 8,6 & 4,2 & 18,8 & 7,3 & 2,6 \\
\hline 30 a 34 & 39,5 & 11,3 & 3,5 & 22,3 & 9,6 & 2,3 \\
\hline 35 a 39 & 47,0 & 16,1 & 2,9 & 29,8 & 14,7 & 2,0 \\
\hline 40 a 44 & 60,9 & 23,4 & 2,6 & 41,2 & 20,2 & 2,0 \\
\hline 45 a 49 & 81,3 & 35,7 & 2,3 & 61,5 & 27,3 & 2,3 \\
\hline 50 a 59 & 131,5 & 65,4 & 2,0 & 89,5 & 43,2 & 2,1 \\
\hline 60 a 69 & 276,5 & 153,8 & 1,8 & 198,4 & 108,6 & 1,8 \\
\hline 70 ou mais & 756,3 & 575,2 & 1,3 & 518,6 & 384,5 & 1,3 \\
\hline
\end{tabular}

Fonte: IBGE - Censo Demográfico de 2000 e 2010; Fundaçăo Seade.

(") Indice de Sobre Mortalidade Masculina. 
mulheres, em favor dessas últimas, que vinha aumentando e atingiu seu máximo em 2000, quando chegou a 9 anos, diminuiu para 7,2 anos, em 2010 (Tabela 1).

Entre 1960 e 2010, a esperança de vida aos dez anos de idade aumentou 6,5 anos para os homens e 9,4 anos para as mulheres. Conforme mostra a Tabela 1, esses ganhos foram maiores entre os homens (3,8 anos) do que entre as mulheres (2,1 anos) no período mais recente. Assim, a sobremortalidade masculina nessa idade diminuiu de 8,9 anos, em 2000, para 7 anos, em 2010. A Tabela 2 ilustra esse fato, mostrando que, em 2000, a mortalidade masculina entre 15 e 29 anos de idade era mais de quatro vezes superior à feminina; em 2010, essa diferença reduziu-se para menos de três vezes. Depois dos 45 anos, o diferencial por sexo não se modificou em todo o período estudado.

Este artigo objetiva avaliar, para o Estado de São Paulo, as repercussões sobre o tempo que a população permanece na atividade econômica, devido as mudanças ocorridas nos níveis e estrutura da mortalidade por sexo, idade e causa de morte, entre 2000 e 2010.

\section{MATERIAIS E MÉTODOS}

Para a realização desse estudo, são utilizadas informações dos censos demográficos realizados pelo IBGE em 2000 e 2010 e as estatísticas de óbitos provenientes do Sistema de Estatísticas Vitais, elaborado pela Fundação Seade. Calculam-se as taxas de atividade brutas e específicas, por sexo e idade, e o número de anos de vida ativa, utilizando as tábuas de mortalidade construídas para esses anos. São também estimadas a esperança de vida aos dez anos de idade, na hipótese de eliminar grupos de doenças classificadas como evitáveis ${ }^{4}$, e sua repercussão no tempo de permanência na atividade econômica.

Este artigo está dividido em quatro itens, além desta introdução. O primeiro se refere à vida ativa do paulista, no período 2000-2010. O segundo, apresenta estimativas do número de anos na atividade econômica, sem e com a influência da mortalidade. No terceiro, analisam-se os efeitos das causas de morte no tempo de permanência na atividade econômica e, finalmente no quarto, apresenta-se um provável cenário dessa participação, ao se eliminar determinadas causas de morte.

\footnotetext{
${ }^{4}$ Segundo Rutstein et. al. (1976), as mortes evitáveis são aquelas que poderiam ter sido evitadas, total ou parcialmente, com serviços de saúde efetivos (causas evitáveis).
} 


\section{RESULTADOS E DISCUSSÃO}

\subsection{VIDA ATIVA DO PAULISTA}

No Estado de São Paulo, os níveis de participação masculina nas atividades econômicas são sistematicamente mais elevados do que os das mulheres, para todas as idades ativas, em 2000 e 2010 . Entretanto, enquanto nesse período a participação masculina apresentou redução de mais de 3\%, a feminina registrou aumento superior a $9 \%$. A maior participação masculina ocorre entre as idades de 30 a 39 anos (acima de $90 \%$ ) e a de mulheres encontra-se na faixa de 25 a 34 anos.

Por sua vez, as taxas de atividade dos mais jovens (menores de 20 anos), idades mais diretamente relacionadas com a estrutura econômico-social e as políticas governamentais, tiveram queda tanto entre os homens como entre as mulheres, mais acentuada no sexo masculino. Entre os homens, a variação dessas taxas continua sendo negativa até os 45 anos, enquanto para as mulheres a variação positiva é cada vez mais intensa ao se avançar na idade, superando $60 \%$ entre aquelas de 60 anos e mais (Gráfico 1).

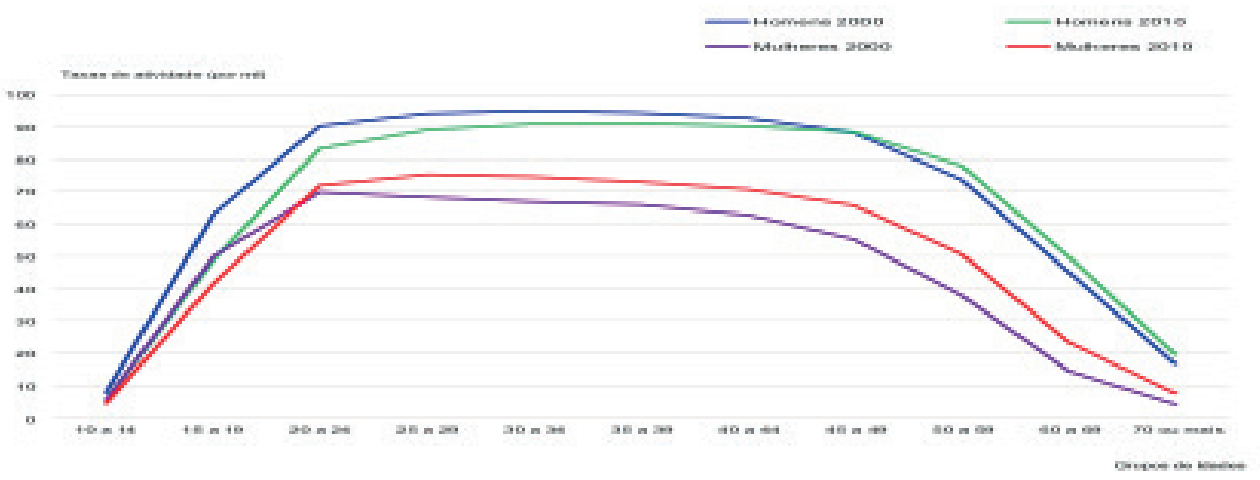

Gráfico 1 - Taxas de atividade, segundo sexo Estado de São Paulo - 2000-2010

Fonte: IBGE. Censos Demográíicos de 2000 e 2010.

No estudo da PEA, um dos aspectos mais importante é conhecer a duração média da vida ativa de uma geração, ou seja, identificar o número médio de anos que se espera que um indivíduo permanecerá em atividade, na hipótese de que as taxas de participação não se modifiquem nesse período. Uma forma de acompanhar essa geração é calcular o número bruto de anos de vida, 
indicador fortemente influenciado pela idade de entrada e saída da atividade econômica do indivíduo e pelas taxas de atividade nessas idades. A mortalidade somente incidiria após a idade-limite de saídas. Assim, esse indicador reflete a permanência e a integração da população na PEA, em ausência de mortalidade.

Por sua vez, o número líquido de anos de vida ativa representa o número médio de anos economicamente ativos de uma geração que em atividade sofresse também os efeitos da mortalidade ${ }^{5}$.

Em São Paulo, em ausência de mortalidade, em média um trabalhador do sexo masculino ficaria em atividade por praticamente 45 anos, em um potencial máximo possível de 70 anos, se todas as pessoas fossem economicamente ativas dos 10 aos 80 anos. Entre as mulheres, esse número aumentou de 28 anos, em 2000, para 31 anos, em 2010. Entretanto, mesmo com esse incremento da participação feminina, o número de anos brutos de atividade masculina é 40\% maior que o de atividade feminina, fato que ilustra as enormes dificuldades da mulher para incorporar-se ao mercado produtivo (Tabela 3).

\section{TABELA 3}

Número bruto de anos de vida ativa, por sexo
Estado de São Paulo - 2000 e 2010

\begin{tabular}{|c|c|c|c|c|}
\hline \multirow{3}{*}{$\begin{array}{c}\text { Grupo de } \\
\text { Idades (anos) }\end{array}$} & \multicolumn{4}{|c|}{ Número Bruto de Anos de Vida Ativa } \\
\hline & \multicolumn{2}{|c|}{ Homens } & \multicolumn{2}{|c|}{ Mulheres } \\
\hline & 2000 & 2010 & 2000 & 2010 \\
\hline 10 a 19 & 3,6 & 2,7 & 2,9 & 2,3 \\
\hline 20 a 29 & 9,2 & 8,6 & 6,9 & 7,4 \\
\hline 30 а 39 & 9,5 & 9,1 & 6,6 & 7,4 \\
\hline 40 a 49 & 9,1 & 9,0 & 5,9 & 6,8 \\
\hline 50 a 59 & 7,4 & 7,8 & 3,8 & 5,1 \\
\hline 60 a 69 & 4,5 & 5,0 & 1,4 & 1,6 \\
\hline $\begin{array}{l}70 \text { e }+ \\
\text { TOTAL }\end{array}$ & $\begin{array}{c}1,7 \\
44,9\end{array}$ & $\begin{array}{l}2,0 \\
44,1\end{array}$ & $\begin{array}{c}0,4 \\
\mathbf{2 8 , 0}\end{array}$ & $\begin{array}{c}0,7 \\
31,3\end{array}$ \\
\hline
\end{tabular}

Fonte: IBGE - Censo Demográfico de 2000 e 2010; Fundação Seade.

Chama atenção o fato de que, no caso dos homens e, em menor medida, entre as mulheres, em 2010, o número estimado de anos na atividade econômica fique acima do número mínimo de anos de contribuição à previdência para a aposentadoria integral, estabelecido, pela Previdência Social, em 35 anos para os homens e 30 para as mulheres. Esses resultados podem estar sugerindo que parte considerável da população encontra-se à margem desses benefícios e/ ou que uma parcela significativa desse segmento tenha retornado à atividade econômica depois da aposentadoria ${ }^{6}$.

\footnotetext{
${ }^{5}$ Pode-se considerar, de certa forma, que a diferença entre anos de vida ativa brutos e líquidos é similar à registrada entre as taxas bruta e líquida de reprodução, como medidas de fecundidade e renovação da população.

${ }^{6}$ Na prática, essa permanência é menor pelo efeito redutor da mortalidade, que pode ocorrer antes do termo do período de atividade econômica. Essa redução é maior para os homens pelo fato de a mortalidade masculina ser mais elevada do que a feminina, especialmente a partir dos dez anos de idade.
} 
Por idade, a situação é bastante diferencial com relação ao gênero. Se considerada, por exemplo, a situação da faixa etária de 30 a 39 anos, na qual as taxas de participação masculina e feminina são mais elevadas, verifica-se que, em média, um trabalhador do sexo masculino ficará 9,1 anos em atividade, dos 10 anos possíveis, enquanto uma trabalhadora ficará somente 7,4 anos. É interessante ressaltar que, entre os homens com idade inferior a 50 anos, o número de anos na atividade econômica diminui entre 2000 e 2010, enquanto acima dessa idade aumenta. Já entre as mulheres registra-se aumento a partir dos 20 anos de idade.

\subsection{O NÍVEL DE MORTALIDADE}

O "número líquido de anos de vida ativa" leva em conta os efeitos diferenciais da mortalidade, por idade e sexo, isto é, considera a influência da mortalidade, sendo que o efeito redutor dessa variável na atividade será dado pela diferença entre os dois indicadores: vida bruta e vida líquida.

Para estimar os anos líquidos, são utilizadas as tábuas de mortalidade construídas para o Estado de São Paulo, centradas nos anos de 2000 e 2010. O índice resultante, que contempla a variável mortalidade, é diretamente comparável à esperança de vida (ex) dá tábua de mortalidade.

Comparando o número de anos brutos e líquidos, tem-se uma medida da influência da mortalidade no número de anos potencialmente ativos. Se examinada inicialmente a situação para os homens, é possível notar que o efeito mortalidade reduz significativamente o tempo de permanência na vida ativa: $17,2 \%$ em 2000 e 11,9\% em 2010. Já entre as mulheres, a redução no número de anos na vida ativa por causa da mortalidade é bem menor: chega a 6,5\% em 2000 e é nula em 2010.

Por sua vez, quando comparados os anos líquidos de vida ativa com a esperança de vida ao início da atividade econômica, pode-se ter uma estimativa dos anos potencialmente inativos, possibilitando avaliar o grau de utilização da mão de obra, de acordo com o gênero. Observa-se que a inatividade potencial masculina aos dez anos aumenta de 19,3 para 23,2 anos, enquanto a feminina registra uma redução de 39,5 para 38,3 anos, mas ainda é $65 \%$ superior à masculina. Isto é, a inatividade masculina, que em 2000 representava 50,4\% do número de anos ativos, aumentou para 58,9\% em 2010. Entre as mulheres, em 2000, a inatividade representava $150 \%$ a mais do que os anos de vida ativa, proporção que diminuiu para 121,8\% em 2010 (Tabela 4). 


\section{TABELA 4}

\section{Efeito da mortalidade na vida ativa}

Estado de São Paulo - 2000 e 2010

\begin{tabular}{|c|c|c|c|c|c|c|c|}
\hline \multirow{2}{*}{ Sexo } & \multicolumn{2}{|c|}{$\begin{array}{c}\text { Número de Anos de Vida } \\
\text { Ativa aos } 10 \text { anos } \\
\end{array}$} & \multirow{2}{*}{$e_{10}^{\circ}(3)$} & \multirow{2}{*}{$(1)-(2)$} & \multirow{2}{*}{$\begin{array}{c}{[(1)-(2)] /} \\
(2)(\%)\end{array}$} & \multirow{2}{*}{$\begin{array}{l}\text { (3) - (2) } \\
\text { (anos) }\end{array}$} & \multirow{2}{*}{$\begin{array}{l}{[(3)-(2)] \prime} \\
(2)(\%)\end{array}$} \\
\hline & Brutos (1) & Liquidos (2) & & & & & \\
\hline $\begin{array}{l}\text { Homens } \\
\text { Mulheres }\end{array}$ & $\begin{array}{l}44,9 \\
28,0\end{array}$ & $\begin{array}{l}38,3 \\
26,3 \\
\end{array}$ & $\begin{array}{l}57,6 \\
65,8 \\
\end{array}$ & $\begin{array}{l}6,6 \\
1,7 \\
\end{array}$ & $\begin{array}{c}17,2 \\
6,5 \\
\end{array}$ & $\begin{array}{l}19,3 \\
39,5 \\
\end{array}$ & $\begin{array}{c}50,4 \\
150,3 \\
\end{array}$ \\
\hline \multicolumn{8}{|l|}{2010} \\
\hline $\begin{array}{l}\text { Homens } \\
\text { Mulheres }\end{array}$ & $\begin{array}{l}44,1 \\
31,3\end{array}$ & $\begin{array}{l}39,4 \\
31,4\end{array}$ & $\begin{array}{l}62,6 \\
69,7\end{array}$ & $\begin{array}{c}4,7 \\
-0,1\end{array}$ & $\begin{array}{l}11,9 \\
-0,3\end{array}$ & $\begin{array}{l}23,2 \\
38,3\end{array}$ & $\begin{array}{c}58,9 \\
121,8\end{array}$ \\
\hline
\end{tabular}

Fonte: IBGE - Censos Demográficos de 2000 e 2010; Fundaçăo Seade.

Esses resultados possibilitam inferir o tempo não dedicado à atividade econômica, motivado pelo retiro profissional, seja pela aposentadoria, voluntária ou não, seja pela entrada mais tarde no mercado de trabalho. Uma consequência desse processo pode ser observada no fato de que os homens têm entrado mais tarde e saído mais cedo da atividade econômica. Já as mulheres, ainda que registrem diminuição no número de anos de inatividade potencial, apresentam uma perda $65 \%$ mais elevada do que a dos homens $(38,3$ contra 23,2 anos), pela soma do efeito do retiro profissional precoce, que ocorre pelo casamento ou pela fecundidade, com os fatores que afetam também a população masculina (ALVES; CORREIA, 2009).

\subsection{CAUSAS DA MORTALIDADE}

Dada a magnitude das saídas da atividade econômica por morte, analisa-se, a seguir, a situação da mortalidade adulta no Estado de São Paulo. A forma de agrupamento das causas de morte, assume grande importância para identificar os fatores que afetam a mortalidade e, sua classificação de acordo com a possibilidade que se tem de preveni-los, representa o primeiro passo para um melhor conhecimento a respeito dos determinantes da mortalidade.

Assim, foi utilizada uma classificação que agrupa as mortes em evitáveis e não evitáveis, adotando-se como mortes evitáveis "as causas de morte cuja evitabilidade é dependente de tecnologia disponível no Brasil, de tecnologia acessível pela maior parte da população brasileira ou de tecnologia ofertada pelo Sistema Único de Saúde" (MALTA et al., 2007b) ${ }^{7}$.

A Tabela 5 mostra a distribuição de óbitos de pessoas entre 10 e 69 anos de idade, por sexo, segundo as causas de morte. Em 2000, pouco mais de $70 \%$ dos 
óbitos registrados nessa população, em ambos os sexos, ocorreram em razão de alguma das doenças consideradas evitáveis na atualidade, proporção que diminui no período em análise, principalmente para a população masculina. Observa-se, também, uma ligeira redução das causas "mal definidas", enquanto aumenta expressivamente as mortes consideradas "não claramente evitáveis", que em 2010 chegavam a representar mais de 20\% dos óbitos dessa faixa etária.

\section{TABELA 5 \\ Distribuição proporcional dos b́bitos de 10 a 69 anos de idade, por sexo, segundo grupos de causas de morte \\ Estado de São Paulo - 2000 e 2010}

\begin{tabular}{|c|c|c|c|c|}
\hline \multirow{2}{*}{ Grupos de Causas de Morte } & \multicolumn{2}{|c|}{ Homens } & \multicolumn{2}{|c|}{ Mulheres } \\
\hline & 2000 & 2010 & 2000 & 2010 \\
\hline $\begin{array}{l}\text { TOTAL } \\
\text { 1. Causas evitaveis por acōes adequadas de }\end{array}$ & 100,0 & 100,0 & 100,0 & 100,0 \\
\hline $\begin{array}{l}\text { promoção à saude, prevenção, controle e } \\
\text { atenção: }\end{array}$ & 76,4 & 72,8 & 70,1 & 68,9 \\
\hline 1.1. Açōes de imunoprevenção & 0,2 & 0,1 & 0,2 & 0,0 \\
\hline 1.2. As doenças de causas infecciosas & 9,2 & 10,0 & 10,1 & 11,4 \\
\hline 1.3. As doenças não transmissiveis & 37.1 & 41,8 & 50,9 & 50,1 \\
\hline 1.4. As causas de morte materna & 0,0 & 0,0 & 0,7 & 0,6 \\
\hline 1.5. As causas externas & 29,9 & 21,0 & 8,2 & 6,8 \\
\hline 2. Causas mal definidas & 6,4 & 5,9 & 5,9 & 4,5 \\
\hline 3. Demais causas (nāo claramente evitáveis) & 17,2 & 21,3 & 24,2 & 26,6 \\
\hline
\end{tabular}

Fonte: Fundação Seade.

O principal grupo de causas de morte é aquele que engloba as mortes reduzíveis por ações adequadas de promoção à saúde, prevenção, controle e atenção às doenças não transmissíveis: para os homens, verifica-se um aumento de sua importância relativa, passando de $37,1 \%$ para 41,8\%, entre 2000 e 2010; entre as mulheres, essas causas superam os $50 \%$, permanecendo constante no período. Segundo a OMS (2013), as doenças não transmissíveis são as principais causas de enfermidade e morte prematura passíveis de prevenção nas Américas.

O substancial ônus socioeconômico que tais doenças acarretam, sobretudo o acentuado aumento nos gastos de tratamento, prejudica o bem-estar de indivíduos e famílias e ameaça travar o avanço socioeconômico. As ações centram-se nas doenças cardiovasculares (DCV), câncer, diabetes e doenças respiratórias crônicas, "bem como em seus quatro fatores de risco comuns, a saber: consumo de tabaco, dieta insalubre, sedentarismo e uso prejudicial de álcool; e os fatores de risco biológicos a elas relacionadas: a hipertensão arterial e os níveis elevados de glicose no sangue" (OMS, 2013). 
Estudo feito pelo Ministério da Saúde em 2002 (BRASIL, 2005) mostrou que as doenças não transmissíveis são responsáveis pelas maiores proporções de anos de vida perdidos por morte prematura $(59,0 \%)$, por anos de vida vividos com incapacidade $(74,7 \%)$ e por anos de vida perdidos ajustados por incapacidade $(66,3 \%)$ (DALY).

Os dados mostram que, em São Paulo, em 2010, na faixa etária de 10 a 69 anos, entre as causas de morte que formam esse grupo, aquelas relacionadas a doenças isquêmicas do coração e doenças cerebrovasculares representaram mais de $40 \%$ dos óbitos entre os homens e 36\% entre as mulheres. $\mathrm{O}$ diabetes mellitus, nos homens, e as neoplasmas da mama, nas mulheres, responderam por mais de $6 \%$ das mortes desse grupo.

Os óbitos por doenças reduzíveis por ações intersetoriais adequadas de promoção à saúde, prevenção e atenção às causas externas, que entre os homens constituem o segundo grupo em importância relativa, registraram expressiva redução entre 2000 e 2010 , passando de $29,9 \%$ para $21,0 \%$, entre os homens, e de $8,2 \%$ para $6,8 \%$, entre as mulheres. As causas específicas de maior peso relativo são as agressões, que, em 2010, representaram 27,8\% das mortes, para a população masculina, e 19,5\%, para a feminina.

As mortes do grupo de doenças reduzíveis por ações adequadas de promoção à saúde, prevenção, controle e atenção às doenças de causas infecciosas, em ambos os sexos, responderam por cerca de $10 \%$ das mortes. No período 2000 2010, estes óbitos registraram tendência diferenciada segundo gênero: uma ligeira redução entre os homens e um incremento que supera os $25 \%$ entre as mulheres.

Neste grupo, as mortes por infecções respiratórias, inclusive pneumonia e influenza, e as doenças pelo vírus HIV são as de maior peso relativo.

Dado o perfil diferenciado de causas de morte por sexo, enquanto as taxas de mortalidade da população masculina de 10 a 69 anos decresceram 13\%, as taxas femininas aumentaram mais de $11 \%$. Entre os homens, $58 \%$ da redução da mortalidade nessa faixa etária foi ocasionada pela diminuição das mortes do grupo de "ações adequadas de promoção à saúde, prevenção, controle e atenção às doenças não transmissíveis”. Entre a população feminina, tais causas foram responsáveis por mais de $73 \%$ do aumento de óbitos registrado no período, nessa faixa etária (Tabela 6). 
TABELA 6

Taxas de mortalidade da populaçăo de 10 a 69 anos de idade, por sexo, segundo grupos de causas de morte

Estado de Săo Paulo - 2000 e 2010

Por 100 mil hab

\begin{tabular}{|c|c|c|c|c|c|c|}
\hline \multirow[b]{2}{*}{ Grupos de Causas de Morte } & \multicolumn{3}{|c|}{ Homens } & \multicolumn{3}{|c|}{ Mulheres } \\
\hline & 2000 & 2010 & $\begin{array}{c}\text { Variação } \\
(\%)\end{array}$ & 2000 & 2010 & $\begin{array}{c}\text { Variação } \\
(\%)\end{array}$ \\
\hline TOTAL & 614,5 & 533,8 & $-13,1$ & 249,3 & 278,5 & 11,7 \\
\hline $\begin{array}{l}\text { 1. Causas evitáveis por açðes adequadas } \\
\text { de promoçăo à saúde, prevençăo, controle e } \\
\text { atençăa: }\end{array}$ & 469,3 & 388,4 & $-17,2$ & 174,4 & 191,9 & 10,0 \\
\hline 1.1. Açס̄es de imunoprevençăa & 1,0 & 0,3 & $-70,3$ & 0,4 & 0,1 & $-70,9$ \\
\hline 1.2. As doenças de causas infecciosas & 56,3 & 53,4 & $-5,2$ & 25,1 & 31,7 & 26,2 \\
\hline 1.3. As doenças năo transmissiveis & 228,0 & 222,9 & $-2,2$ & 127,0 & 139,4 & 9,8 \\
\hline 1.4. As causas de morte matema & NA & NA & NA & 1,8 & 1,8 & $-1,2$ \\
\hline 1.5. As causas externas & 183,9 & 111,8 & $-39,2$ & 20,5 & 19,0 & $-7,2$ \\
\hline 2. Causas mal definidas & 39,5 & 31,6 & $-20,1$ & 14,7 & 12,6 & $-14,0$ \\
\hline 3. Demais causas (não claramente evitáveis) & 105,7 & 113,7 & 7,6 & 60,2 & 74,0 & 22,8 \\
\hline
\end{tabular}

Fonte: Fundaçăo Seade.

As mortes do grupo de causas reduzíveis por “ações Intersetoriais adequadas de promoção à saúde, prevenção e atenção às causas externas” apresentaram significativo decréscimo no período analisado e foram responsáveis por $28,9 \%$ da queda da mortalidade masculina de 10 a 69 anos; já entre as mulheres, essa contribuição representou $9,8 \%$.

No período, observa-se significativa redução nas mortes classificadas como "mal definidas". O grupo de causas classificadas como "não claramente evitáveis" registrou aumento para ambos os sexos: $7,6 \%$ na população masculina e $22,8 \%$ na feminina.

\subsection{UM PROVÁVEL CENÁRIO}

O método estatístico que permite estudar situações nas quais os indivíduos estão expostos ao risco de morrer por várias causas é conhecido como análise de riscos competitivos, sendo que um dos instrumentos dessa análise é a tábua de mortalidade de múltiplo decremento. Em termos de riscos competitivos, considera-se que um indivíduo, durante sua vida, está exposto ao risco de morrer por diversas causas, independentemente da causa que definitivamente ocasionou sua morte. Nesse sentido, entende-se que houve uma certa “competição" entre os vários riscos. É importante, então, diferenciar os conceitos de risco e causa. Embora ambos sejam da mesma natureza, o termo 
risco refere-se ao fenômeno antes do óbito, a partir do qual se torna a causa (CHIANG, 1968).

Por meio desse estudo, pode-se determinar qual seria o efeito, na vida média da população, da eliminação e/ou redução da intensidade de determinada doença ou causa. Também é possível conhecer a taxa de mortalidade devido a uma causa específica, na presença de outras causas de morte (CHIANG, 1968; ORTIZ 2000).

A eliminação dos óbitos do grupo de mortes reduzíveis por ações adequadas de promoção à saúde, prevenção, controle e atenção às doenças não transmissíveis aumentaria em 2,6 anos a esperança de vida da população masculina aos dez anos de idade. Para a população feminina, esse incremento seria de 1,3 ano. Na hipótese de eliminar as mortes do grupo reduzíveis por ações Inter setoriais adequadas de promoção à saúde, prevenção e atenção às causas externas, os ganhos seriam de 1,7 e 0,2 ano, respectivamente, para homens e mulheres. Se fossem eliminadas todas as doenças consideradas evitáveis na atualidade, o acréscimo seria de 5,3 anos, para os homens, e de 2,8 anos, para as mulheres (Tabela 7).

\section{TABELA 7}

Esperança de vida aos 10 anos de idade, por sexo, segundo grupos de causas de morte Estado de São Paulo - 2010

Em anos

\begin{tabular}{|c|c|c|c|c|c|}
\hline \multirow{2}{*}{ Grupos de Causas de Morte } & \multicolumn{3}{|c|}{ Esperança de vida aos 10 anos } & \multicolumn{2}{|c|}{ Ganhos } \\
\hline & Homens & Mulheres & Diferença & Homens & Mulheres \\
\hline Situaçao atual & 62,6 & 69,6 & 7,1 & & \\
\hline \multicolumn{6}{|l|}{ Eliminação hopotética de: } \\
\hline 1. Todas as doenças evitáveis & 67,9 & 72,4 & 4,5 & 5,3 & 2,8 \\
\hline 1.1. Açōes de imunoprevenç̧̃̃o & 62,6 & 69,6 & 7,0 & 0,0 & 0,0 \\
\hline 1.2. As doenças de causas infeccloses & 63.2 & 69,9 & 6,7 & 0,6 & 0,3 \\
\hline 1.3. As doenças não transmissiveis & 65,2 & 70,9 & 5,7 & 2,6 & 1,3 \\
\hline 1.4. As causas de morte materna & 62,6 & 69,6 & 7,0 & 0,0 & 0,0 \\
\hline 1.5. As causas externas & 64,3 & 69,8 & 5,5 & 1,7 & 0,2 \\
\hline 2. Demais causas (nalo claramente evitáveis) & 63,9 & 70,3 & 6,4 & 1,3 & 0,7 \\
\hline
\end{tabular}

Fonte: Cálculos do autor.

Cabe salientar que, como esperado, a eliminação das causas de morte afeta mais significativamente a esperança de vida da população masculina do que a da feminina, com consequências nos diferenciais por gênero. Trata-se de um resultado coerente, dado que o padrão de causas de morte está associado a inserções ocupacionais diferenciadas por sexo, o que determina a maior exposição dos homens ao risco de morte. 
A sobremortalidade masculina, que na média chega a 7,1 anos, reduziria para 4,5 anos na hipótese de serem eliminadas todas as causas evitáveis, e para 5,5 anos, no caso de se eliminarem as mortes do grupo de reduzíveis por ações Inter setoriais adequadas de promoção à saúde, prevenção e atenção às causas externas.

\section{CONSIDERAC̣ÕES FINAIS}

No período 2000-2010, no Estado de São Paulo, a participação da população economicamente ativa masculina permaneceu praticamente constante - em torno de $70 \%$-, enquanto a PEA feminina registrou contínuos e significativos aumentos, superando os $52 \%$ em 2010, o que estaria fortemente relacionada com as mudanças econômicas e sociais ocorridas no Brasil e, em particular, em São Paulo, nas últimas décadas (ALVES; CORREIA, 2009).

A situação de estabilidade para a população masculina estaria ocorrendo por conta de uma permanência maior dos jovens na escola, fenômeno que vem adiando seu ingresso no mercado de trabalho, enquanto entre as pessoas mais idosas o aumento da cobertura previdenciária contribui para retirá-las mais cedo da PEA. Nesse sentido, alguns estudos indicam redução da participação dos jovens que estudam e trabalham e um leve aumento para os que não estudam e não trabalham (MENEZES FILHO, et. al.2013).

Estudo de IPEA (2013), mostra que "esse aumento da inatividade dos jovens pode ainda se tornar um fator limitador para futuros ganhos de produtividade da economia”. Esta situação poderia ocorrer devido a que esse contingente populacional "não estão nem se qualificando nem adquirindo experiência no mercado de trabalho". Esse mesmo estudo indica que, no caso da população feminina, o expressivo aumento da taxa de participação é reflexo de profundas mudanças sociais e políticas na sociedade brasileira, bem como das mudanças significativas nas relações familiares, em que, por exemplo, ganha cada vez mais importância relativa a presença de uma chefe mulher.

$\mathrm{O}$ intenso processo de envelhecimento da população paulista, reflexo da elevada queda na taxa de fecundidade, associada ao aumento da expectativa de sobrevida e de uma forte redução da migração, acentuado de maneira significativa na última década, tem como reflexo uma redução significativa da razão entre ativos e inativos. Esse processo, aliado a outros fatores, tem provocado aumento relativo de doenças não transmissíveis e das causas externas, que incidem principalmente na população em idades ativas e com impacto sobre os gastos com saúde. Esta situação se torna aguda pelo fato de 
ocorrer num quadro em que ainda persiste o desafio de aprimorar a vigilância e o controle das doenças infecciosas.

Em São Paulo, as várias estratégias implementadas, como as de vigilância e controle de doenças imunopreveníveis, têm sido fundamentais para redução ou erradicação, por exemplo da poliomielite, o sarampo, a difteria, o tétano e a coqueluche. No entanto, as mortes prematuras evitáveis e o envelhecimento populacional exigem mudanças expressivas de organização do sistema de saúde, privilegiando as ações de promoção e prevenção das doenças crônicas e de promoção da saúde.

$\mathrm{Na}$ última década, no Estado, a mortalidade masculina por idade mostrou uma redução muito mais acentuada do que a feminina, revertendo o aumento da sobremortalidade masculina que se observava desde 1960. Como resultado desse processo, a influência da mortalidade no tempo de permanência na atividade econômica perde intensidade, mas, ainda assim, a inatividade masculina em relação ao número de anos ativos aumentou no período, chegando a representar praticamente $60 \%$, em 2010, enquanto a feminina, embora reduzindo-se, alcançou mais de $120 \%$.

Esse quadro pode ser atenuado se for possível reduzir e/ou eliminar doenças que ocorrem pela falta de ações adequadas às doenças não transmissíveis e por causas externas. Nessa situação, o diferencial segundo gênero da mortalidade seria reduzido significativamente, bem como aumentariam expressivamente a esperança de vida aos dez anos de idade e, consequentemente, o tempo de permanência na atividade econômica, especialmente para a população masculina. 


\section{REFERÊNCIAS}

ALVES, J. E. D.; CORREA, S. Igualdade e desigualdade de gênero no Brasil: um panorama preliminar, 15 anos depois do Cairo. Brasil, 15 anos após a Conferência do Cairo. Campinas: Abep/UNFPA, 2009. BRASIL. Ministério da Saúde. A vigilância, o controle e a prevenção das doenças crônicas não transmissíveis - DCNT - no contexto do Sistema Único de Saúde brasileiro. Brasilia: Organização Pan-Americana da Saúde, 2005.

CAMARANO, A. A.; KANSO, S. 0 que estão fazendo os jovens que não estudam, não trabalham e não procuram trabalho?

Boletim Mercado de Trabalho, v. 53, nov. 2012.

CHIANG, CL. Introduction to stocastic processes in biostatistics. 1968, New York: John Wiley \& Sons.

IPEA - Instituto de Pesquisa Econômica Aplicada. Efeitos da População Economicamente Ativa sobre a taxa de desemprego. Nota Técnica. Carta de Conjuntura, dez. 2013.

MALTA, D.; DUARTE, E. Causas de morte evitáveis por ações efetivas dos serviç,os de saúde: uma revisão da literatura. Ciência e Saúde Coletiva, v.12, n³, 2007a.

MALTA, D. et. al. Lista de causas de mortes evitáveis por intervenções do Sistema Único de Saúde do Brasil. Epidemiol. Serv. Saúde, v. 16, n $n^{0} 4,2007 b$.

MENEZES FILHO, N A. et. al. A condição "nem-nem" entre os jovens é permanente? Insper, 2013. (Policy Paper, n 7).

OMS - Organização Mundial da Saúde. Plano de ação para a prevenção e controle de doenças não transmissíveis. Washington, D.C.: 520 Conselho Diretor. $65^{\circ}$ Sessão do Comitê Regional, 2013.

ORTIZ. L. P. Mortalidade por causas evitáveis no Estado de São Paulo - 1975/1976. Informe Demográfico, Fundação Seade, $n^{0}$ 4, 1984.

. The competing risk study of infant mortality Brazilian. Journal of Population Studies. Associação Brasileira de Estudos de População - Abep, 2000.

RUSTSTEIN DD, et.al. Measuring the quality of medical care: a clinical method. The New England Journal of Medicine 1976; 294(11).

YAZAKL, L. Estatísticas de nascimentos: mães mais velhas e crescimento desenfreado de cesáreas em São Paulo. SP Demográfico, ano 13, n² 2, março 2013.

WALDVOGEL, B. et.al. Mudanças nos componentes da dinâmica demográfica paulista. SP Demográfico, ano 12, $n^{0}$ 3, outubro 2012. 